\title{
Nuevos Conceptos sobre la Prevención de la Hipocalcemia en Ganado Lechero
}

\author{
Melendez, $P$.
}

Colegio de Medicina Veterinaria, Universidad de Georgia, EEUU. 501. D.W. Brooks Drive, Athens, GA 30602

Correo electrónico: pedro.melendezp@uga.edu

\section{Resumen}

La fiebre de leche o hipocalcemia continúa siendo una de las enfermedades metabólicas de mayor prevalencia en la industria lechera, como consecuencia a una continua selección de los animales por pro-ductividad. En la presente revisión se describen nuevos aspectos de la enfermedad, interacciones minerales, tratamientos y medidas preven-tivas bajo confinamiento y en pastoreo.

Palabras claves: fiebre de leche, calcio, dietas aniónicas, ganado lechero

\section{The new concept of the hypocalcemia prevention on dairy cattle}

\section{Abstract}

Milk fever or hypocalcemia still is one of the most prevalent metabolic diseases as a consequence of the continued selection for the productivity of the dairy cattle. The present manuscript reviews the new aspect of the disease, mineral interactions, treatments and preventive measurement under either cattle in confinement or on pasture.

Key words: milk fever, calcium, anionic salts, dairy cattle

Fecha de recepción artículo original: 10/06/2018

Fecha de aceptación para su publicación: 20/11/2018 


\section{Desarrollo}

La fiebre de leche o hipocalcemia clínica es una enfermedad metabólica típica del ganado lechero que se caracteriza por una deficiencia aguda de calcio en la sangre lo que conlleva a una serie de manifestaciones clínicas, incluyendo la muerte del animal. Los signos clínicos van a depender del grado de deficiencia de calcio que por lo general ocurren entre las 24 a 48 horas después de parida la vaca(1)

El calcio (Ca) es un macromineral importante que desempeña un papel central en el mantenimiento de la vida de los vertebrados. El Ca es un elemento fundamental en la contracción muscular, la coagula-ción sanguínea, la actividad enzimática, la excitabilidad neuronal, la secreción de hormonas, y la adhesión celular, además de ser un componente estructural esencial del esqueleto. El control preciso del ion $\mathrm{Ca}$ en los fluidos extracelulares es vital. Para mantener una concentra-ción constante de $\mathrm{Ca}$ han evolucionado mecanismos de control endo-crino que consisten principalmente en la interacción de tres hormo-nas principales: la hormona paratiroidea (PTH), la calcitonina (CT) y la vitamina D (Vit D) ${ }^{(1)}$ Las acciones de la PTH son (i) aumentar la tasa de conversión de la pre-vitamina 25-(OH) D3 (Hidroxicolecalciferol) a 1, 25 $(\mathrm{OH})_{2}$ D3 (Di-hidroxicolecalciferol) en el tejido renal, (ii) aumen-tar las concentraciones de Ca en plasma; (iii) incrementar la extensión de la osteólisis osteoclástica y osteocítica en el hueso (reabsorción y remodelación ósea); y (iv) aumentar la excreción urinaria de fosfatos y disminuir la excreción urinaria de Ca. Los efectos biológicos clásicos de la vitamina $\mathrm{D}$ activa son: (i) estimular la absorción de calcio y fós-foro de la dieta en el intestino; (ii) promover la mineralización de la matriz ósea y estimular la reabsorción ósea en los huesos; (iii) reducir la excreción urinaria de fosfato y Ca en el riñón. $(2,3,4)$

Durante el periparto, es inevitable cierta reducción de los niveles de Ca en la sangre (hipocalcemia) de la vaca lechera. En algunas vacas, la reducción es lo suficientemente grande como para ser considerada significativa para la salud y se denomina hipocalcemia subclínica. Se caracteriza por una concentración de calcio en sangre $<2.0 \mathrm{mmol} / \mathrm{L}(<8.0 \mathrm{mg} / \mathrm{dl})$. ${ }^{(5)}$ Sin embargo, últimamente este valor de corte se ha incrementado a $2,15 \mathrm{mmol} / \mathrm{L}(8,5 \mathrm{mg} / \mathrm{dl})^{(6)}$

La hipocalcemia se desarrolla como resultado del rápido drenaje de Ca al calostro durante el inicio de la lactancia, lo que resulta en un tremendo desafío para la capacidad de la vaca en mantener los niveles normales de Ca. ${ }^{(1)}$ De hecho, en un estudio recientemente publicado se ha visto que los niveles de Ca se reducen en sangre al menos 9 horas antes del inicio del parto en vacas que han sido alimentadas con dietas 
aniónicas.(8) Entre un 10 a un $50 \%$ de las vacas pueden desarrollar niveles bajos de Ca (hipocalcemia subclínica), pero sin signos de hipocalcemia clínica, incluso hasta 10 días después del parto. ${ }^{(8,5,9,1)}$ La hipocalcemia puede afectar los órganos que presentan tejido muscular liso, como el útero, el rumen, el abomaso, y el esfínter del pezón. En consecuencia, la hipocalcemia es un factor de riesgo importante para las distocias, la retención de membranas fetales, metritis, prolapso uterino, desplazamiento del abomaso, mastitis, cetosis clínica e hígado graso.(10,11,12) Estos trastornos se han asociado con una mala expresión del celo (estro), quistes ováricos y metritis, que afectan negativamente el rendimiento reproductivo de las vacas lecheras. ${ }^{(13,14)}$

Cuando se desarrolla una hipocalcemia clínica ( $\mathrm{Ca}<5 \mathrm{mg} / \mathrm{dl}$ ), generalmente es fácil de tratar, pero aumenta en forma considerable la probabilidad de enfermedades secundarias. Por lo tanto, la prevención de esta condición metabólica es esencial para el éxito del período de transición y el resto de la lactancia. Una estrategia preventiva exitosa involucra la manipulación de la diferencia entre aniones y cationes de la dieta (DCAD), que se ha definido como la diferencia en miliequiva-lentes de cationes (principalmente Na y K) y aniones (principalmente $\mathrm{S}$ y $\mathrm{Cl}$ ) por kilogramo de materia seca (MS), lo que tiene un impacto directo en el metabolismo ácido-base de la sangre $(15,16,4)$ Se han utili-zado varios métodos para calcular la DCAD y las ecuaciones que se han propuesto son las siguientes(4)

$\operatorname{DCAD}(\mathrm{mEq})=(\mathrm{Na}+\mathrm{K})-(\mathrm{Cl}+\mathrm{S})$

$\operatorname{DCAD}(\mathrm{mEq})=(\mathrm{Na}+\mathrm{K})-(\mathrm{Cl})$

$\operatorname{DCAD}(\mathrm{mEq})=(\mathrm{Na}+\mathrm{K}+0.15 \mathrm{Ca}+0.15 \mathrm{Mg})-(\mathrm{Cl}+0.2 \mathrm{~S}+0.3 \mathrm{P})$

$\operatorname{DCAD}(\mathrm{mEq})=(\mathrm{Na}+\mathrm{K}+0.15 \mathrm{Ca}+0.15 \mathrm{Mg})-(\mathrm{Cl}+0.25 \mathrm{~S}+0.5 \mathrm{P})(4)$

$\operatorname{DCAD}(\mathrm{mEq})=(\mathrm{Na}+\mathrm{K})-(\mathrm{Cl}+0.6 \mathrm{~S})$

A pesar de no ser la más precisa, debido a que no considerara otros iones importantes en la regulación del metabolismo ácido-base, la ecuación que más comúnmente se ha utilizado es la (1): DCAD $(\mathrm{mEq})=(\mathrm{Na}+\mathrm{K})-(\mathrm{Cl}+\mathrm{S})$.

Las dietas con un DCAD negativo inducen una acidosis metabólica compensada y reducen el riesgo de fiebre de leche ${ }^{(17,3,1)}$ A medida que disminuye el DCAD, aumentan los $\mathrm{H}^{+}$en la sangre, disminuye $\mathrm{HCO}^{-3}$ y disminuye el $\mathrm{pH}$. Estos cambios están acompañados por una reduc-ción en la excreción urinaria de $\mathrm{Na}$ y $\mathrm{K}$ y una reducción en el pH uri-nario como mecanismos compensatorios. El mecanismo que explica 
la reducción en el grado de hipocalcemia es que la acidosis moderada incrementa la capacidad de respuesta de los tejidos a las hormonas homeostáticas que controlan los niveles de Ca en $\operatorname{sangre}^{(15,17,4)}$ Las dietas típicas ofrecidas a vacas secas tienen un DCAD de aproximadamente $+50 \mathrm{a}+250 \mathrm{mEq} / \mathrm{kg}$ MS. En los forrajes, el $\mathrm{K}$ es el mineral más variable de los iones en la ecuación de DCAD y generalmente es el que más contribuye a aumentar el DCAD en la dieta y conlleva a que la vaca antes del parto esté en un estado de alcalosis metabólica compensa$\mathrm{da}^{(3,18,4)}$ El uso exitoso de aniones dietéticos para prevenir la fiebre de la leche ha sugerido que las dietas ricas en cationes, especialmente Na y $\mathrm{K}$, aumentan la susceptibilidad de las vacas a la fiebre de la leche. En un estudio clásico, se determinó el efecto de la adición de $\mathrm{K}$ o Na interactuando con niveles bajos y altos de $\mathrm{Ca}$ en las dietas preparto. Este estudio demostró que el Ca dietético (0.5 o 1.5\%) no afectó significativamente la incidencia de fiebre de leche o el grado de hipocalcemia experimentado por las vacas, sin embargo, el aumento de $\mathrm{K}(2.1$ o $3.1 \%)$ y $\mathrm{Na}(1.3 \%)$ en la dieta, amplificó la incidencia de la fiebre de la leche. El $\mathrm{pH}$ de la sangre y la orina aumentaron y la hidroxiprolina en plasma se redujo mediante la adición de cationes fuertes. Esto sugirió que la reabsorción ósea de Ca se inhibía en las vacas alimentadas con alto $\mathrm{K}$ o Na como resultado de la alcalosis metabólica ${ }^{(2)}$. Por lo tanto, el nivel de calcio en la dieta no es el factor más importante en determinar la aparición de hipocalcemia, sino que los niveles de cationes, sobre todo el potasio. A la luz de los resultados, muchos nutricionistas, incluido el autor de este artículo, prefieren ofrecer dietas aniónicas con una concentración de Ca en la dieta de entre 0.8 a 1.0\%.

La fuerte relación negativa $\left(\mathrm{r}^{2}=0,95\right)$ que existe entre el $\mathrm{pH}$ urina-rio y la excreción ácida neta de las vacas alimentadas con dietas que contienen sales aniónicas sugiere que la medición del pH urinario es una herramienta útil para evaluar el grado de acidosis metabólica que impone la dieta aniónica.(17) Una ventaja de este enfoque es que no depende de las inexactitudes en los análisis de minerales y los cam-bios inesperados en el contenido mineral de forraje. Por ejemplo, los valores de $\mathrm{pH}$ urinario por debajo de 5,5 indican un riesgo de sobre acidificación y el establecimiento de una acidosis metabólica no com-pensada. ${ }^{(1,19)} \mathrm{El}$ pH urinario óptimo es entre 6.0 y 7.0 para las vacas Holstein y entre 5.8 y 6.2 para las vacas Jersey.(1) En los rebaños le-cheros con una incidencia alta de fiebre de leche ( $>2 \%$ ), la orina de las vacas preparto debe ser muy alcalina, con un $\mathrm{pH}$ superior a 8.0. Sin embargo, los resultados más exactos se obtienen mediante la recolec-ción de muestras de orina a una hora estándar, preferiblemente den-tro de unas pocas horas después de haber alimentado a las vacas. $(20,4)$ 
Los compuestos basados en cloruros causan una mayor acidificación de la orina que los compuestos basados en sulfato, a igual equivalencia o número de cargas. (21)

En un estudio de metaanálisis se demostró que al reducir el DCAD a un nivel de $0 \mathrm{mEq} / \mathrm{kg}$ MS y el $\mathrm{pH}$ de la orina de alrededor de 7.0, la incidencia de fiebre de leche se redujo en forma dramática. También se vio que los niveles de Ca en sangre y la incidencia de hipocalcemia clínica no se redujeron en forma mucho más significativa cuando el DCAD se llevó por debajo de $-100 \mathrm{mEq} / \mathrm{kg}$ MS o el $\mathrm{pH}$ de la orina $<6.0^{(20)}$

Hay pocos estudios que demuestren que alimentar con un DCAD negativo durante más de 40 días perjudicaría el rendimiento de la vaca lechera en transición y su descendencia. ${ }^{22,23)}$ Hasta ahora, la eviden-cia científica propone alimentar un DCAD entre 0 y - $100 \mathrm{mEq} / \mathrm{kg}$ MS durante un periodo de no más de 28 días y alcanzar un $\mathrm{pH}$ en orina entre 6.0 a 7.0. Los posibles trastornos y daños que podemos inducir en la fisiología de la vaca y el feto a largo plazo debido a un exceso de aniones por un periodo prolongado de tiempo no se conocen. De he-cho, se ha argumentado que la inducción de una acidosis metabólica más severa ( $\mathrm{pH}$ de la orina $<6.0$ ) puede llevar a la vaca a un estado de acidosis metabólica no compensada con graves consecuencias para el animal y el conceptus ${ }^{(1)}$. Debemos recordar que la escala de $\mathrm{pH}$ es logarítmica y cuando bajamos el pH de 8 a 7 estamos incrementando los protones en orina en 10 veces. Cuando bajamos el $\mathrm{pH}$ a 6 , estamos incrementando los protones en 100 veces y cuando disminuimos el $\mathrm{pH}$ a 5, lo estamos haciendo en 1000 veces. En base a esta elucida-ción, la sobrecarga del riñón para excretar el exceso de protones en 1000 veces, con el objeto de mantener el $\mathrm{pH}$ de la sangre dentro de los rangos normales, es más que evidente e irrefutable. Por otro lado, se indican frecuentemente dietas preparto con un DCAD por debajo de -100 (cerca de - $140 \mathrm{mEq} / \mathrm{kg} \mathrm{MS}$ ), no obstante, después de una revi-sión profunda de la literatura científica, no se ha encontrado evidencia que demuestre que disminuir el $\mathrm{pH}$ de la orina por debajo de 6.0 y el DCAD por debajo de $-100 \mathrm{mEq} / \mathrm{kg}$ MS, se traduzca en un beneficio notable para la vaca durante el postparto temprano. La mejor eviden-

cia científica que respalda esta afirmación es el metaanálisis realizado por Charbonneau et al. (2006) ${ }^{(20)}$ En ese estudio, la reducción del DCAD de +300 a $0 \mathrm{mEq} / \mathrm{kg}$ MS y $\mathrm{pH}$ de la orina de 8.1 a 7.0 redujo significativamente la incidencia de fiebre de leche de $16.4 \%$ a $3.2 \%$, pero también disminuyó el consumo de MS en un $11 \%$. Este último punto es importante de considerar, ya que producto de la poca palatabilidad de las sales aniónicas, una reducción en el consumo puede llevar a la vaca preparto a movilizar grasa antes de tiempo y por ende aumentar 
los riesgos de otras enfermedades metabólicas.(12) La reducción del DCAD por debajo de $-100 \mathrm{mEq} / \mathrm{kg}$ MS y el $\mathrm{pH}$ de la orina por debajo de 6.0 no mejoró significativamente la homeóstasis del Ca y la inciden-cia de fiebre de la leche. Además, en un estudio reciente que comparó dietas preparto con un DCAD + $165 \mathrm{mEq} / \mathrm{kg}$ MS versus un DCAD - $138 \mathrm{mEq} / \mathrm{kg}$ MS no se observaron diferencias en las concentraciones de Ca plasmático y la incidencia de hipocalcemia.(23) Sin embargo, otros estudios reportaron que vacas Holstein con un $\mathrm{pH}$ urinario por debajo de 6,0 tuvieron concentraciones de Ca en sangre al momento del par-to entre 1,9 y $1,95 \mathrm{mmol} / \mathrm{L}$, a pesar de que el DCAD preparto fue de $-150 \mathrm{y}$ $-200 \mathrm{mEq} / \mathrm{kg}$ DM. ${ }^{(18,24)}$ Otro estudio reportó que vacas con un $\mathrm{pH}$ urinario de alrededor de 6.5 durante el preparto tuvieron concentraciones de $\mathrm{Ca}<2.0 \mathrm{mmol} / \mathrm{L}$ hasta 48 horas posparto después de que se alimentaron con una dieta preparto con un DCAD de $-170 \mathrm{mEq} / \mathrm{kg}$ DM. ${ }^{(7)}$ Estos hallazgos sugieren que hay varios factores involucrados en la homeostasis del $\mathrm{Ca}$, incluida la concentración de $\mathrm{Ca}, \mathrm{P}$ y $\mathrm{Mg}$ en la ración durante el período preparto.(16) De hecho, en un estudio realizado en Nueva Zelanda, las dietas experimentales ofrecidas a vacas lecheras preparto con un DCAD que varió de $+23 \mathrm{a}+88 \mathrm{mEq} / 100 \mathrm{~g}$ de MS, dieron como resultado un pH urinario que varió entre 7.93 y 8.36, pero las concentraciones totales de Ca en plasma durante la primera semana postparto oscilaron entre 2.48 y $2.59 \mathrm{mmol} / \mathrm{L}$. ${ }^{(25)}$

Para complementar los supuestos de que un exceso de acidificación no beneficia al animal, el grupo de medicina productiva de la Universidad de Missouri decidió llevar a cabo un estudio de campo donde se tomó una muestra de sangre en aproximadamente 10 vacas al momento del parto que fueron alimentadas con una dieta con un DCAD de $-140 \mathrm{mEg} / \mathrm{kg}$ MS. Luego se cambió la dieta preparto a una con un DCAD de $-50 \mathrm{mEq} / \mathrm{kg}$ MS y se muestrearon otras 10 vacas al momento del parto. Después de cambiar la estrategia nutricional para las vacas preparto se vio que el Ca total fue similar entre ambos gru-pos, con un valor promedio de alrededor de $8,5 \mathrm{mg} / \mathrm{dL}$, que es el punto de corte para la hipocalcemia subclínica.(19) Esto demostró que no es necesario bajar el DCAD más allá de $-100 \mathrm{mEq} / \mathrm{kg}$ MS para incremen-tar las concentraciones de Ca plasmático. En la Tabla 1 se resumen las dietas ofrecidas a las vacas en este estudio. En la Tabla 2 se repor-ta la composición nutricional de las dietas. En la Tabla 3 se detallan las mediciones de $\mathrm{pH}$ urinario antes y después del cambio de dieta y en la Tabla 4 se resumen los parámetros metabólicos de las vacas muestreadas. 
Tabla 1. Dietas preparto (kg/vaca/dia)

\begin{tabular}{|l|c|c|c|c|}
\hline \multicolumn{1}{|c|}{ Ingredientes } & \multicolumn{2}{c|}{ DCAD -143 $\mathrm{mEq} / \mathrm{kg}$} & \multicolumn{2}{c|}{ DCAD -53 $\mathrm{mEq} / \mathrm{kg}$} \\
\hline & TCO & MS & TCO & MS \\
\hline Heno Brome & 5.81 & 5.0 & 2.5 & 2.23 \\
\hline Ensilaje de maíz & 8.34 & 2.72 & 14.5 & 4.73 \\
\hline Maíz grano molido fino & 2.58 & 2.27 & 0.8 & 0.7 \\
\hline Premezcla preparto DCAD & 1.56 & 1.43 & - & - \\
\hline Premezcla preparto DCAD & - & - & 1.6 & 1.42 \\
\hline Harina Soja 47.5\% Solvente & 1.30 & 1.18 & 1.2 & 1.06 \\
\hline Cascara de Soja & 0.49 & 0.45 & 2.5 & 2.27 \\
\hline Cebada húmeda & - & - & 4.0 & 0.98 \\
\hline \multicolumn{1}{|c|}{ Total } & 20.12 & 13.07 & 27.1 & 13.42 \\
\hline
\end{tabular}

TCO: tal cual ofrecido, MS: materia seca

Tabla 2. Composición Nutricional dietas preparto

\begin{tabular}{|c|c|c|}
\hline Nutriente & DCAD -140 mEq/kg MS & DCAD -50 mEq/kg MS \\
\hline Materia Seca (\%) & 64.9 & 49.5 \\
\hline Proteina Cruda (\%) & 14.01 & 14.51 \\
\hline Proteina Soluble (\%) & 3.18 & 3.51 \\
\hline PND \# $(\%)$ & 5.56 & 6.51 \\
\hline aFDNom $^{\ddagger}(\%)$ & 44.79 & 44.11 \\
\hline peFDN \& $(\%)$ & 36.77 & 32.2 \\
\hline Almidon (\%) & 19.1 & 17.42 \\
\hline $\mathrm{CNF}^{\$}(\%)$ & 28.16 & 26.5 \\
\hline Grasa (\%) & 2.79 & 2.89 \\
\hline $\mathrm{Ca}(\%)$ & 1.31 & 0.94 \\
\hline $\mathrm{P}(\%)$ & 0.30 & 0.34 \\
\hline $\mathrm{Mg}(\%)$ & 0.41 & 0.47 \\
\hline $\mathrm{K}(\%)$ & 1.10 & 1.13 \\
\hline $\mathrm{Na}(\%)$ & 0.072 & 0.09 \\
\hline $\mathrm{S}(\%)$ & 0.28 & 0.26 \\
\hline $\mathrm{Cl}(\%)$ & 1.0 & 0.78 \\
\hline $\mathrm{Cu}(\mathrm{ppm})$ & 23 & 14 \\
\hline Se (ppm) & 0.28 & 0.26 \\
\hline Zn (ppm) & 53 & 51 \\
\hline Co (ppm) & 0.76 & 0.70 \\
\hline DCAD (mEq $/ \mathrm{kg})^{*}$ & -143 & -53 \\
\hline
\end{tabular}

${ }^{*}$ DCAD formula $(\mathrm{Na}+\mathrm{K})-(\mathrm{Cl}+\mathrm{S})$

\# Proteina No Degradable ${ }^{\ddagger}$

FND libre cenizas

\& FDN fisicamente efectiva

\$Carbohidratos No Fibrosos 
Tabla 3. Media y Rango $\mathrm{pH}$ Urinario

\begin{tabular}{|l|c|c|}
\hline \multicolumn{1}{|c|}{ Fecha } & Media & Rango \\
\hline Periodo con DCAD -143 mEq/kg MS & & \\
\hline Octubre 21, 2015 & 5.48 & $5.32-5.71$ \\
\hline Noviembre 11, 2015 & 5.53 & $5.11-5.89$ \\
\hline Diciembre 16, 2015 & 5.81 & $5.41-6.12$ \\
\hline Enero 20, 2016 & 5.72 & $5.53-6.20$ \\
\hline Febrero 24, 2016 & 5.70 & $5.53-5.91$ \\
\hline Periodo con DCAD -50 mEq/kg MS & & \\
\hline Marzo 23, 2016 & 6.67 & $5.70-8.23$ \\
\hline Marzo 30, 2016 & 6.90 & $6.11-8.23$ \\
\hline Abril 06, 2016 & 6.58 & $5.88-7.53$ \\
\hline
\end{tabular}

Tabla 4. Resultados de laboratorio de vacas al parto alimentadas con una DCAD -143 y DCAD -50

\begin{tabular}{|l|c|c|c|c|}
\hline \multicolumn{1}{|c|}{ Metabolito } & $\begin{array}{c}\text { DCAD -143 } \\
(\mathrm{n}=10)\end{array}$ & $\begin{array}{c}\text { DCA -50 } \\
(\mathrm{n}=11)\end{array}$ & P-value & $\begin{array}{c}\text { Valores } \\
\text { Referencia }\end{array}$ \\
\hline Calcio (mg/dL) & $8.45 \pm 0.91$ & $8.44 \pm 0.64$ & 0.50 & $8.0-11.0$ \\
\hline Fosforo (mg/dL) & $4.51 \pm 1.3$ & $5.47 \pm 1.63$ & 0.16 & $4.3-8.0$ \\
\hline Magnesio (mg/dL) & $2.45 \pm 0.23$ & $2.60 \pm 0.44$ & 0.35 & $2.0-3.5$ \\
\hline Sodio (mEq/L) & $143.3 \pm 2.66$ & $143.9 \pm 5.14$ & 0.74 & $137-148$ \\
\hline Potasio (mEq/L) ** & $5.38 \pm 0.91$ & $6.32 \pm \pm 1.74$ & 0.14 & $3.8-5.2$ \\
\hline Cloro (mEq/L) & $106 \pm 2.26$ & $106.1 \pm 4.45$ & 0.95 & $97-111$ \\
\hline Albumina (g/dL) & $3.34 \pm 0.17$ & $3.49 \pm 0.23$ & 0.93 & $3.0-3.6$ \\
\hline Globulina (g/dL) $* *$ & $2.98 \pm 0.36$ & $3.22 \pm 0.74$ & 0.35 & $3.7-3.9$ \\
\hline Creatinina (mg/dL) & $1.078 \pm 0.12$ & 1.0710 .15 & 0.92 & $1.0-2.0$ \\
\hline $\begin{array}{l}\text { Gamma glutamil } \\
\text { transferasa (IU/L) }\end{array}$ & $12.6 \pm 2.27$ & $14.2 \pm 5.89$ & 0.21 & $13-33$ \\
\hline $\begin{array}{l}\text { Creatina Fosfokinasa } \\
\text { (IU/L) }\end{array}$ & $1124.1 \pm 1839$ & 399.5249 .9 & 0.21 & $105-409$ \\
\hline
\end{tabular}

Otro punto importante por analizar es que la evaluación de las dietas aniónicas en ganado lechero preparto en condiciones de pastoreo no ha sido estudiada en forma consistente. Las dietas basadas en pas-turas, que a menudo son ricas en $\mathrm{K}$, pueden comprometer la capacidad de los suplementos aniónicos para inducir efectivamente una acidosis metabólica leve en la vaca. Incluso la alimentación de una ración par-cial con sales aniónicas, junto con el pastoreo, es un desafío grande debido a las variaciones en la cantidad de $\mathrm{K}$ y pasto consumido. Por lo tanto, el grupo de medicina productiva de Missouri decidió llevar ade-lante un estudio para comparar el efecto de dos dietas aniónicas mix-tas parciales (PMR) basadas en sulfatos o cloruros. Un grupo de vacas 
recibió una dieta en base a sulfato de magnesio y el otro grupo de animales recibió una dieta en base a ácido clorhídrico. En ambos grupos la mitad de la MS fue aportada por una ración completa con las fuen-tes minerales aniónicas y la otra mitad fue aportada pastoreo (Festuca spp.). El pH promedio de la orina fue menor en las vacas alimentadas con cloruros en relación con aquellas alimentadas con sulfatos, en cuatro de las cinco ocasiones de muestreo (Tabla 5). Las concentracio-nes promedio de Ca en plasma en el día 1 fueron de 2.00 (IC 95\% = 1.94$2.05)$ y 1.91 (IC $95 \%=1.88-1.97$ ) $\mathrm{mmol} / \mathrm{L}$ para los grupos que recibieron cloruros y sulfatos, respectivamente $(\mathrm{p}=0.15)$, y al día 3 fue de 2.03 (IC $95 \%=1.97-2.08$ ) y 1.90 (IC $95 \%=1.84-1.96$ ) $\mathrm{mmol} / \mathrm{L}$, respectivamente $(p=0.038)$. Las concentraciones de $\mathrm{Mg}$, P y BHB en plasma y la incidencia de enfermedades posparto fueron similares en ambos grupos y no hubo casos de hipocalcemia clínica en ninguno de los grupos. Se concluyó que las vacas preparto alimentadas con una ra-ción mixta parcial con un DCAD negativo que contiene sales aniónicas basadas en cloruros y el acceso libre a una pradera de festuca tuvieron un $\mathrm{pH}$ de orina preparto más bajo y concentraciones de Ca más altas en plasma durante la primera semana postparto, en comparación con vacas que recibieron una ración mixta parcial con sales aniónicas en base a sulfatos. Lo interesante del estudio es que la raza utilizada fue una cruza Jersey con Holstein, que tiene un componente genético de una raza mucho más susceptible a desarrollar cuadros de hipocalce-mia, como es la Jersey. No obstante, con la dieta en base a cloruros se logró mejorar en forma significativa la concentración de Ca al día del parto y además no se registraron casos de fiebre de leche, lo cual es el elemento más importante en el objetivo del uso de dietas aniónicas. (26)

Cuando se siguen todas las reglas del punto de vista del uso de las sales aniónicas, y cuando se logra reducir el pH urinario a los niveles deseados de entre 6,0 y 6,8, aun algunos predios lecheros pueden presentar una mala experiencia con la fiebre de leche. Esta inconsisten-cia se puede asociar lo más probable a algún grado de deficiencia de magnesio, sobre todo en animales a pastoreo, que consumen prade-ras muy tiernas, ricas en potasio y en nitrógeno. ${ }^{(4)}$ De hecho, la hipomagnesemia, o tetania de los pastos, es un cuadro típico de animales a pastoreo en épocas de otoño e invierno. El cuadro se caracteriza por animales postrados en el suelo, que expresan movimientos bruscos de sus miembros, y que los ganaderos describen como que si los animales estuvieran "pataleando" o "pedaleando".

El magnesio es un macromineral esencial para la vida que tiene muchos roles, pero uno de los más importantes se relaciona a la función del sistema nervioso y actividad de las neuronas. Cuando 
ocurre una deficiencia de magnesio, se produce una hiperexcitabilidad de las neuronas, generando un cuadro nervioso y muscular típico, con movimientos bruscos de los miembros, que finalmente puede llevar a una falla cardio-respiratoria y muerte del animal. ${ }^{(1)}$

Tabla 5. pH urinario de vacas preparto manejadas a pradera

\begin{tabular}{|l|c|c|c|}
\hline \multicolumn{1}{|c|}{ Date } & $\begin{array}{c}\text { Dieta en base } \\
\text { a Sulfatos }\end{array}$ & $\begin{array}{c}\text { Dieta en base } \\
\text { a Cloruros }\end{array}$ & P-value \\
\hline $\begin{array}{l}\text { Agosto 19, 2016, } \\
\text { pre-experimento }\end{array}$ & $8.27 \pm 0.25$ & $8.38 \pm 0.23$ & 0.20 \\
\hline Agosto 23, 2016 & $8.56 \pm 0.18$ & $8.26 \pm 0.22$ & $<0.01$ \\
\hline Septiembre 01, 2016 & $7.87 \pm 0.08$ & $7.85 \pm 0.10$ & 0.54 \\
\hline Septiembre 07, 2016 & $8.13 \pm 0.08$ & $7.94 \pm 0.13$ & $<0.01$ \\
\hline Septiembre 14, 2016 & $7.81 \pm 0.10$ & $7.72 \pm 0.12$ & 0.06 \\
\hline Al parto & $7.76 \pm 0.12$ & $7.34 \pm 0.13$ & $<0.01$ \\
\hline
\end{tabular}

La concentración normal de magnesio en el plasma de las vacas lecheras debe ser entre 1,8 a 2,4 $\mathrm{mg} / \mathrm{dl}(0,75$ a $1,0 \mathrm{mmol} / \mathrm{L})$. El mantenimiento del magnesio sanguíneo dentro de concentraciones normales es prácticamente dependiente de un aporte constante de magnesio a partir de la dieta. El magnesio se absorbe con mayor eficiencia en el in-testino delgado de terneros jóvenes, pero no así en bovinos adultos. Esto se explica porque a medida que se desarrolla el rumen y el retículo, es-tos órganos se convierten en el principal, y tal vez el único sitio, para la absorción neta de magnesio hacia la sangre. La absorción de magnesio desde el rumen depende de la concentración del mineral en el líquido ruminal y de la integridad del mecanismo de transporte de magnesio a través de las paredes del rumen. El transporte activo de magnesio a través del rumen se hace fundamental cuando su contenido dietario no se encuentra en cantidades suficientes. Desafortunadamente, una alta concentración de $\mathrm{K}$ en la dieta y en el fluido ruminal conlleva a que se despolarice la membrana apical del epitelio ruminal, reduciendo el mecanismo de absorción activo de magnesio a través de la pared del rumen. Por lo tanto, una ración que puede parecer normal del punto de vista del contenido de magnesio puede llegar a ser deficitaria en magnesio cuando el potasio dietario es excesivo ${ }^{(1,4)}$ típico de las praderas en crecimiento activo de temporadas de otoño e invierno. Existe una segunda vía para la absorción del magnesio sin que se vea afectada por el contenido de $\mathrm{K}$ de la dieta, y que se basa en un transporte pasivo de este mineral. Lamentablemente, este proceso de transporte pasivo solo opera con altas concentraciones de magnesio en el fluido del rumen, lo que permite al magnesio moverse a través de un gradiente de 
concentración desde el lugar de mayor (el fluido del rumen) a un lugar de menor concentración (los fluidos extracelulares de las paredes del rumen). La concentración de magnesio en el fluido ruminal necesario para lograr una gradiente de concentración y una absorción pasiva del mineral debe ser mayor a $4 \mathrm{mmol} / \mathrm{L}$. Por lo tanto, el nivel mínimo de magnesio requerido en la dieta para evitar los efectos negativos de un exceso de $\mathrm{K}$ en los rumiantes es de aproximadamente 0,35\%. Así, el contenido de magnesio de la ración de una vaca preparto y de una vaca postparto debe ser de entre 0,35 a $0,45 \%$ (4)

Se debe tener en cuenta que al usar oxido de magnesio como fuente única, puede que no sea lo suficientemente soluble como para proporcionar la cantidad necesaria que requiere la vaca. Hay una amplia variación en la biodisponibilidad del magnesio proveniente de diferentes fuentes de óxido de magnesio, por lo tanto, el muestreo de magnesio sanguíneo, dentro de las 12 horas de paridas, proporciona un índice efectivo del estado de este mineral en la vaca periparturienta. Si la concentración de magnesio en suero $\leqslant 1,8 \mathrm{mg} / \mathrm{dl}(0,8 \mathrm{mmol} / \mathrm{L})$, indica que la dieta es inadecuada en su contenido y absorción de magnesio y por lo tanto esa hipomagnesemia puede limitar la productividad y a su vez contribuir al desarrollo de hipocalcemia en el rebaño. ${ }^{4)}$

La hipomagnesemia afecta el metabolismo del calcio de dos maneras: 1) al reducir la secreción de la hormona PTH en respuesta a la hipocalcemia y 2) al reducir la sensibilidad de los tejidos a la hormona PTH. La integridad de la interacción entre la PTH y sus receptores en los tejidos, sobre todo en los huesos, es vital para la homeostasis del calcio. La hipomagnesemia, independiente de un estado metabólico de alcalosis, también puede interferir con la capacidad de la PTH para actuar sobre sus receptores en los tejidos blancos (hueso, por ejemplo). La evidencia de campo sugiere que las concentraciones de magnesio en la sangre por debajo de $0,65 \mathrm{mmol} / \mathrm{L}$ en la vaca periparturienta aumentará la susceptibilidad de las vacas a la hipocalcemia subclínica y a la fiebre de leche. Por lo tanto, la concentración soluble de magnesio en el fluido ruminal va a depender de: (i) el contenido de magnesio en la dieta, y (ii) el pH del rumen: la solubilidad del magnesio disminuye bruscamente a medida que aumenta el pH del rumen por sobre 6,5. En ciertas ocasiones, los animales en pastoreo tienen un $\mathrm{pH}$ ruminal más alto debido al alto contenido de $\mathrm{K}$ de los pastos y la estimulación de la salivación asociada al pastoreo, con aporte de bicarbonato. Además, los pastos fuertemente fertilizados a menudo tienen un alto contenido de nitrógeno no proteico y relativamente bajo en carbohidratos solu-bles de fácil fermentación. Por lo tanto, va a haber un exceso de amonio en el rumen, lo que también van a aumentar su pH. Existen también 
quelantes del magnesio en el forraje: los forrajes a menudo contienen un alto contenido (100 a $200 \mathrm{mmol} / \mathrm{kg}$ ) de ácidos grasos insaturados, como el ácido linoleico y linolénico, los que pueden formar sales de magnesio insolubles en el rumen. Las praderas también pueden con-tener ácido trans-aconítico, el cual presenta un metabolito que puede formar un complejo insoluble con el magnesio, que es muy resistente a la degradación del rumen y puede jugar un rol preponderante en la tetania hipomagnesémica de vacas a pastoreo. ${ }^{(4)}$

El magnesio se incluye en las raciones de vacas lecheras para mantener niveles adecuados de magnesio en la sangre y como buffer o alcalinizador del líquido ruminal. El sulfato de magnesio hidratado y el cloruro de magnesio son fuentes de magnesio muy solubles y de muy buena biodisponibilidad, aunque también son relativamente bajos en su contenido de magnesio ( $9 \%$ y 18\%, respectivamente), ya que estas sales de magnesio están altamente hidratadas. También, ellas son muy poco palatables y tampoco actúan como buffers o amortiguadores del $\mathrm{pH}$ en el rumen. El óxido de magnesio (Mg0) es más barato y más palatable. Además, actúa como alcalinizante, por lo que se usa más a menudo como buffer en las dietas de lactancia. Desafortunadamente, hay una gran variabilidad en la biodisponibilidad del magnesio, como oxido. En los rumiantes, el óxido de magnesio se debería ofrecer con un particulado muy fino para tener una mejor biodisponibilidad. Se puede llevar a cabo una prueba de campo para estimar la disponibi-lidad relativa y la calidad de las fuentes de óxido de magnesio a utili-zar: Para tal caso, se debe colocar 3 gramos de la fuente de óxido de magnesio a evaluar en un recipiente con tapa y agregar lentamente $40 \mathrm{ml}$ de ácido acético al $5 \%$ (vinagre blanco). Se debe tapar el envase y agitarlo bien por 15 segundos, y déjelo reposar. Después de 30 minu-tos se debe verificar el $\mathrm{pH}$. El vinagre solo tiene un $\mathrm{pH}$ de 2,6 a 2,8. Las mejores fuentes de óxido de magnesio van a elevar el $\mathrm{pH}$ a 8,2 y las peores fuentes a solo 3,8 .

Finalmente, el uso de productos orales de Ca se ha recomendado al momento del parto para el control y la prevención de la hipocalcemia.

${ }^{(27)}$ Hoy en día se han estado estudiando una serie de suplementos de Ca al momento del parto, en forma de bolos de liberación lenta, independiente si los animales provienen de manejos preparto que utilizan sales aniónicas o se encuentran a pastoreo. Un estudio neozelandés(28) evaluó la administración de dos bolos orales de Ca sobre las concentraciones de Ca total, $\beta$-hidroxibutirato (BHB) y ácidos grasos no esterificados (NEFA) en el suero y pH urinario de vacas lecheras alimentadas a pastoreo durante el preparto. Los dos bolos de Ca se administraron con una diferencia de aproximadamente 12 horas, con 
el primer bolo siendo administrado dentro de las 14 horas posteriores al parto. Cada bolo contenía $43 \mathrm{~g}$ de Ca; de los cuales, $31 \mathrm{~g}$ de Ca provenían a partir de cloruro de calcio y $12 \mathrm{~g}$ a partir de sulfato de calcio. Se tomaron muestras de sangre a las $0,1,2,4,8,12,13,14,16,20$ y 24 horas después del tratamiento inicial. Para el Ca sérico, se observó una interacción entre el tiempo y el tratamiento $(\mathrm{p}=0,004)$, siendo el porcentaje de aumento más alto en las vacas tratadas que en las vacas control a las 1, 2, 4, 8 y 13 horas. A las 12 horas, 5/13 (41\%) de las vacas tratadas tenían un $\mathrm{pH}$ de la orina $<7$ comparado con $0 / 12(0 \%)$ de las vacas de control ( $\mathrm{p}<0,001)$, y a las 24 horas $13 / 13(100 \%)$ de las vacas tratadas tenían un $\mathrm{pH}$ urinario de $<7$ en comparación con $0 / 12(0 \%)$ de las vacas control $(\mathrm{p}<0,001)$. Durante el período de 24 horas, las concentraciones promedio de NEFA o BHB en suero fueron similares entre las vacas tratadas y las de controles. Se concluyó que el tratamiento oral con dos bolos de Ca aumentó las concentraciones de Ca en suero y disminuyó el $\mathrm{pH}$ de la orina en vacas alimentadas a pastoreo. Este bolo tiene el potencial de reducir la prevalencia y la du-ración de la hipocalcemia subclínica en vacas recién paridas.

En un estudio llevado a cabo en un tambo comercial en California se evaluó el efecto de la suplementación oral de Ca sobre el estado mineral de la sangre y algunos indicadores del balance energético en 205 vacas multíparas Jersey bajo estabulación. Después del parto, las vacas se asignaron en forma sistemática y aleatoria al grupo control ( $\mathrm{n}=105$ ) o tratamiento (Sulfato de Ca, aportando 50 a $60 \mathrm{~g}$ de Ca en forma de bolos; $n=100$ ) al momento del parto y al día subsiguiente. La concentración sérica promedio de Ca fue mayor en el grupo tratado que en el grupo control $(2.12$ vs. $2.06 \mathrm{mmol} / \mathrm{L}$, respectivamente). La incidencia de hipocalcemia subclínica fue menor para el grupo tratado que para las vacas controles (53\% vs. $65 \%$, respectivamente); sin em-bargo, a los 2 días postparto la prevalencia de hipocalcemia subclínica tendió a ser mayor para las vacas tratadas. Los autores concluyeron que la concentración sérica de Ca después del parto fue mayor en las vacas tratadas, pero futuros estudios deben considerar evaluar las im-plicancias a largo plazo en la producción de leche y la fertilidad de las vacas Jersey suplementadas con productos orales de Ca de liberación lenta. (29)

Por último, en un estudio llevado a cabo en un tambo del estado de New York se compararon vacas controles, con vacas tratadas con cal-cio subcutáneo o vacas tratadas con un bolo de liberación lenta de Ca. Los autores evaluaron el efecto de estos tratamientos sobre la inciden-cia de metritis, desplazamiento del abomaso, mastitis, fiebre de leche, y retención de membranas fetales. También se evaluaron las tasas de 
descarte, la tasa de concepción al primer servicio y la producción pro-medio leche diaria. Los resultados mostraron que las vacas tratadas en forma subcutánea tuvieron mayores concentraciones de Ca 1 a 12 h después del tratamiento y las vacas que recibieron el bolo oral tuvie-ron mayores concentraciones de Ca 1 y 24 horas post tratamiento en comparación con las vacas controles. No se encontraron diferencias en el riesgo de metritis, desplazamiento del abomaso y las otras con-diciones de salud. Tampoco hubo mejora en la tasa de concepción al primer servicio. La producción de leche fue similar entre los grupos (controles $=46.7 \mathrm{~kg}$; tratamiento subcutáneo $=47.1 \mathrm{~kg}$ y tratamiento con bolos $=47.0 \mathrm{~kg}$ ).

Tampoco hubo diferencias en el riesgo de mastitis entre los tratamientos. Los autores concluyeron que, aunque la suplementación con Ca aumentó las concentraciones de Ca sérico, este efecto no mejoró en gran medida la producción de leche, la fertilidad y los parámetros de salud. ${ }^{(30)}$ A la luz de los resultados de estos 3 experimentos, se ob-serva que los tratamientos con $\mathrm{Ca}$ al parto (bolos de liberación lenta o inyectable) no mejoran en forma consistente el rendimiento produc-tivo de las vacas, siendo quizás los mayores beneficios observados en vacas manejadas bajo pastoreo. La aplicación de Ca inyectable puede en cierta medida generar un efecto negativo o de retroalimentación negativa sobre los mecanismos regulatorios del Ca sanguíneo y por ende se debe ser cuidadoso en suplementar excesos de $\mathrm{Ca}$ al momento del parto. ${ }^{4)}$ Por otro lado, dosis únicas de Ca en forma de tomas orales o "drench" tampoco han demostrado ser efectivas, sobre todo en vacas alimentadas con dietas aniónicas. El incremento en Ca sanguíneo es solo temporal, y las vacas después de un periodo de 6 horas pueden volver a mostrar signos de hipocalcemia subclínica.(31,32)

\section{Conclusión}

En conclusión, el uso de sales aniónicas es una excelente herramienta que ayuda a prevenir la fiebre de leche y enfermedades asociadas, pero requiere de un elevado conocimiento de la nutrición y un eficiente manejo alimentario del rebaño para optimizar sus resulta-dos. El uso de sales aniónicas es una tecnología avanzada que debe ser consistente con el manejo del resto de los minerales y nutrientes de la dieta preparto, sobre todo el magnesio y el potasio. Si no se monitorea en forma rutinaria no se recomienda su uso. El pH urinario se debería medir una vez por semana en un $15 \%$ de las vacas preparto. La orina debe ser limpia y no contaminada buscando lograr un $\mathrm{pH}$ que idealmente oscile entre 6,0 y 7,0. El uso de bolos ruminales de liberación 
lenta de Ca están bajo estudio intensivo en la actualidad por lo tanto no hay resultados concluyentes que recomienden su uso rutinario, de-bido a un costo-beneficio aun relativo. 


\section{Bibliografía}

1. Goff JP. Calcium and magnesium disorders. Veterinary Clinics of. North America Food Animal Practice. 2014; 30: 359-381

2. Goff JP, Horst RL. Effects of the addition of potassium or sodium but not calcium, to prepartum rations on milk fever in dairy cows. Journal of Dairy Science. 1997b; 80: 176-186.

3. Oetzel GR. Management of dry cows for the prevention of milk fever and other mineral disorders. Veterinary Clinics of. North America Food Animal Practice.200; 16:369-386.

4. Goff JP. Invited review: Mineral absorption mechanisms, mineral interactions that affect acid-base and antioxidant status, and diet considerations to improve mineral status. Journal of Dairy Science.2018; 101: 1-51.

5. Reinhardt TA, Lippolis JD, McCluskey BJ, Goff JP, Horst RL. Prevalence of subclinical hypocalcemia in dairy herds. The Veterinary Journal.2011; 188:122-124

6. Martinez N, Risco CA, Lima FS, Bisinotto RS, Greco LF, Ribeiro ES, Maunsell F, Galvão K, Santos JEP. Evaluation of peripartal calcemic status, energetic profile, and neutrophil function in dairy cows at low or high risk of developing uterine disease. Journal of Dairy Science.2012; 95:7158-7172.

7. Megahed AA, Hiew MWH, El Badawy SA, Constable PD. Plasma calcium concentrations are decreased at least 9 hours before parturition in multiparous Holstein-Friesian cattle in a herd fed an acidogenic diet during late gestation. Journal of Dairy Science.2018; 101:1365-1378

8. Melendez P, Donovan GA, Risco CA, Goff JP. Plasma mineral and energy metabolite concentrations in dairy cows fed an anionic prepartum diet that did or did not have retained fetal membranes after parturition. American Journal of Veterinary Research. 2004; 65: 1071-1076.

9. McArt JAA, Nydam DV, Oetzel GR. Epidemiology of subclinical ketosis in early lactation dairy cattle. Journal of Dairy Science.2012; 95:5056-5066

10. Kelton DF, Lissemore KD, Martin RE. Recommendations for recording and calculating the incidence of selected clinical diseases of dairy cattle. Journal of Dairy Science.1998; 81: 2502-2509

11. Melendez P, Risco CA. Management of transition cows to optimize reproductive efficiency in dairy herds. Veterinary Clinic of North America Food Animal Practice.2005; 21:485-501

12. Melendez P, Risco CA.Reproduction, events and management pregnancy: Periparturient disorders. Reference module in food sciences. First ed. Elsevier Academic Press.2016.

13. Goff JP, Horst RL. Physiological changes at parturition and their relationship to meta-bolic disorders. Journal of Dairy Science. 1997a; 80:1260-1268. 
14. Melendez P, Bartolome J. Advances on nutrition and fertility in dairy cattle: Review. Revista Mexicana de Ciencias Pecuarias. Revista Mexicana de Ciencias Pecuarias 2017; 8(4):407-417 http://dx.doi.org/10.22319/rmcp.v8i4.4160

15. Block E. Manipulation of dietary cation-anion difference on nutritionally related production diseases, productivity, and metabolic responses of dairy cows. Journal of Dairy Science. 1994; 77:1437-1450

16. Lean IJ, DeGaris PJ, McNeil DM, Block E. Hypocalcemia in dairy cows: meta-analysis and dietary cation anion difference theory revisited. Journal of Dairy Science.2006; 89: 669-684.

17. Vagnoni DB, Oetzel GR. Effects of dietary cation-anion difference on the acid-base status of dry cows. Journal of Dairy Science.1998; 81:1643-1652

18. Ramos-Nieves JM, Thering BJ, Waldron MR, Jardon PW, Overton TR. Effects of anion supplementation to low-potassium prepartum diets on macromineral status and performance of periparturient dairy cows. Journal of Dairy Science.2009; 92:5677-5691

19. Melendez P, Poock S. A dairy herd case investigation with very low dietary ca-tionanion difference in prepartum dairy cows. Frontiers in Nutrition 2017; 4: 26.

20. Charbonneau E, Pellerin D, Oetzel GR. Impact of lowering dietary cation-anion difference in nonlactating dairy cows: a meta-analysis. Journal of Dairy Science.2006; 89: $537-548$

21. Goff JP, Ruiz R, Horst RL. Relative acidifying activity of anionic salts commonly used to prevent milk fever. Journal of Dairy Science. 2004; 87: 1245-1255.

22. Weich W, Block E, Litherland NB. Extended negative dietary cation-anion difference feeding does not negatively affect postpartum performance of multiparous dairy cows. Journal of Dairy Science. 2013; 96:5780-5792

23. Weiss WP, Azem E, Steinberg W, Reinhardt TA. Effect of feeding 25-hydroxyvitamin D3 with a negative cation-anion difference diet on calcium and vitamin D status of periparturient cows and their calves. Journal of Dairy Science2015; 98:5588-5600.

24. Wu Z, Bernard JK, Zanzalari KP, Chapman JD. Effect of feeding a negative dietary ca-tionanion difference diet for an extended time prepartum on postpartum serum and urine metabolites and performance. Journal of Dairy Science 2014; 97:7133-7143.

25. Roche JR, Petch S, Kay JK. Manipulating the dietary cation-anion difference via drenching to early-lactation dairy cows grazing pasture. Journal of Dairy Science. 2005; 88: 264-276.

26. Melendez P, Zaror V, Gaul P, Poock SE, Goff JP. Effect of diets containing sulfate or chlo-ride-based anionic salts, fed to grazing prepartum dairy cows, on concentrations of $\mathrm{Ca}$ in plasma, disease incidence and milk yield. New Zealand Veterinary Journal.2019. https:// doi.org/10.1080/00480169.2018.1556747

27. Oetzel GR. Oral calcium supplementation in peripartum dairy cows. Veterinary Clinics of. North America Food Animal Practice. 2013; 29:447-455.

28. Roberts KI, Bennison J, McDougall S. 2019. Effect of treatment with oral Ca boluses following calving on concentrations of Ca in serum in pasture-based dairy cows. New Zealand Veterinary Journal. 2019; 67:20-26. doi: 10.1080/00480169.2018.1520654 
29. Valldecabres A, Pires JAAA, Silva-del-Río N. Effect of prophylactic oral calcium supple-mentation on pp mineral status and markers of energy balance of multiparous Jersey cows. Journal of Dairy Science.2018;101: 4460-4472.

30. Domino AR, Korzec HC, McArt JAA. Field trial of 2 calcium supplements on early lactation health and production in multiparous Holstein cows. Journal of Dairy Science. 2017; 100:9681-969.

31. Meléndez P, Donovan A, Risco CA, Hall MB, Littell R, Goff J. Metabolic responses of transition cows fed anionic salts and supplemented at calving with Calcium and Ener-gy. Journal of Dairy Science. 2002; 85:1085-1092

32. Meléndez P, Donovan A, Risco CA, Hall BA, Littell R, Goff J. Effect of calcium-energy supplements on calving-related disorders, fertility and milk yield during the transition period in cows fed anionic diets. Theriogenology. 2003; 60:843-854 